\title{
Grand Challenges in Shape-Changing Interface Research
}

\author{
Jason Alexander \\ Lancaster University \\ Lancaster, UK \\ j.alexander@lancaster.ac.uk \\ Anne Roudaut \\ University of Bristol \\ Bristol, UK \\ roudauta@gmail.com
}

\author{
Jürgen Steimle \\ Saarland University \\ Saarbrücken, Germany \\ steimle@cs.uni-saarland.de
}

\author{
Kasper Hornbæk \\ University of Copenhagen \\ Copenhagen, Denmark \\ kash@di.ku.dk
}

Miguel Bruns Alonso

TU Eindhoven

Eindhoven, The Netherlands

mbruns@tue.nl
Sean Follmer

Stanford University

Palo Alto, CA, USA

sfollmer@stanford.edu
Timothy Merritt

Aalborg University

Aalborg, Denmark

merritt@cs.aau.dk
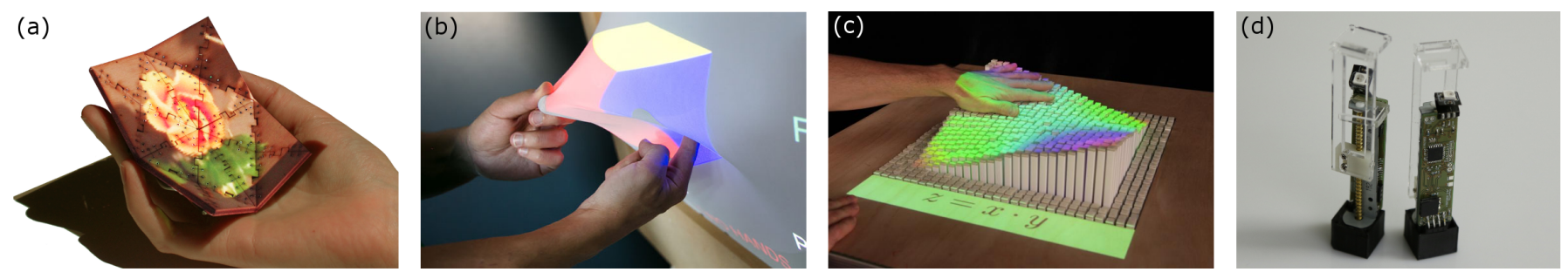

Figure 1. Example shape-changing interfaces: (a) Morphees, a shape-changing mobile phone [75]; (b) An elastic deformable display [102]; (c) inFORM, a self-actuated pin-array [23]; (d) ShapeClip, a prototyping toolkit for shape-changing interfaces [32].

\section{ABSTRACT}

Shape-changing interfaces have emerged as a new method for interacting with computers, using dynamic changes in a device's physical shape for input and output. With the advances of research into shape-changing interfaces, we see a need to synthesize the main, open research questions. The purpose of this synthesis is to formulate common challenges across the diverse fields engaged in shape-change research, to facilitate progression from single prototypes and individual design explorations to grander scientific goals, and to draw attention to challenges that come with maturity, including those concerning ethics, theory-building, and societal impact. In this article we therefore present 12 grand challenges for research on shape-changing interfaces, derived from a threeday workshop with 25 shape-changing interface experts with backgrounds in design, computer science, human-computer interaction, engineering, robotics, and material science.

\section{ACM Classification Keywords}

H.5.2. Information Interfaces and Presentation (e.g. HCI): User Interfaces

\section{Author Keywords}

Shape-changing interfaces; Grand Challenges; Research Agenda.

This work is licensed under a Creative Commons Attribution International 4.0 License.

CHI 2018 April 21-26, 2018, Montreal, QC, Canada

(C) 2018 Copyright held by the owner/author(s).

ACM ISBN 978-1-4503-5620-6/18/04 . . \$15.00

DOI: https : //doi . org/10 . 1145/3173574 . 3173873

\section{INTRODUCTION}

Shape-changing interfaces provide the opportunity to fundamentally transform human interaction with computing machines. Users will change from reading and touching flat glass displays to physically manipulating interfaces that transform their shape and materiality to represent the underlying content and context (see Figure 1). Examples of such interfaces are numerous, and include handheld mobile devices [14, 26], tabletop surfaces [23, 95], furniture [30], and architecture [63]. Each of the examples in the literature are unique, and quite diverse in their goals, fidelity, input/output capabilities, and complexity of construction. However, the overarching challenges faced in their development are often common.

While prior surveys have discussed challenges from a few different disciplinary perspectives $[10,72,91]$, the overarching grand challenges facing the development of shape-changing interfaces have not been systematically explored. With the maturing of the field, it becomes increasingly important to identify a roadmap for future research, as new questions have emerged. These questions result from the acceleration of the technologies for implementing shape-change (e.g. materialsscience, electrical- and mechanical-engineering, bio-science), the increasing number of disciplines involved in shape-change research (e.g. design, psychophysics, philosophy), the increasing number of real-world deployments, and a range of ethical and societal implications that are becoming pertinent as this research grows. In short, the challenges facing the field might both be expanding (through new disciplines and technology) and converging (through the advances in the field).

To help form a research agenda and to give cross-disciplinary researchers exposure to the diverse array of challenges, this article synthesizes the grand challenges that must be addressed 
for shape-changing interfaces to move from vision to successfully embedding themselves in everyday life. These challenges were generated, explored, and distilled during a three-day workshop with 25 experts in shape-changing interfaces, with backgrounds in design, computer science, human-computer interaction, engineering, robotics, and material science.

We envision researchers and practitioners from various fields will use this article to: (1) identify areas of opportunity in the field where they can contribute; (2) situate their work within the larger shape-changing interfaces research agenda; (3) set out new directions for researchers in their own fields; (4) identify current knowledge and capabilities; (5) allow policy makers to better understand the community, state-of-the-art, and potential applications.

\section{WHAT ARE SHAPE-CHANGING INTERFACES? Vision and Definition}

The vision for shape-changing interfaces is for interactive computational devices to transform into any shape or materiality relevant to the context of use. This vision has its roots in Sutherland's Ultimate Display where he describes a computer that can "control the existence of matter" [94]. Shape-changing interfaces change our fundamental approach to interaction design, expanding interactive systems to include our perceptual motor skills to support the same direct interaction our body has with the everyday world. They take advantage of our haptic and kinaesthetic senses, our instinctive perception of physical 3D forms, and provide inherent support for multi-user interaction. More specifically, we define a shape-changing interface to:

1. Use physical change of shape or change in materiality as input and/or output [72].

2. Be interactive and computationally controlled.

3. Be self-actuated [70] and/or user-actuated.

4. Convey information, meaning, or affect.

These scoping statements describe the fundamental nature of a shape-changing interface (1) and are widely inclusive of different forms of interaction (3). We limit this inclusivity to ensure inanimate objects (such as slow-response memory foam) are not included (2) and that these devices must have a purpose or benefit by conveying meaning (e.g. through data, purpose, or affordance) (4).

\section{Describing Shape-Changing Interfaces}

The design space of shape-changing interfaces is vast; to capture this, several taxonomies define elements of shape-change. Coelho and Zigelbaum [10] describe their technological properties, including the power requirements, ability to memorize new shapes, input stimulus or ability to sense deformations. Rasmussen et al. [72] present a review of existing work on shape-changing interfaces, identifying eight types of deformation (orientation, form, volume, texture, viscosity, spatiality, adding/subtracting, permeability). Roudaut et al. [75] propose the term shape-resolution to extend the definition of display resolution to shape-changing interfaces. Finally, Sturdee and Alexander [91] categorise shape-changing interface prototypes with a view to informing their design and application. The reader is referred to these works for full reviews of the field.
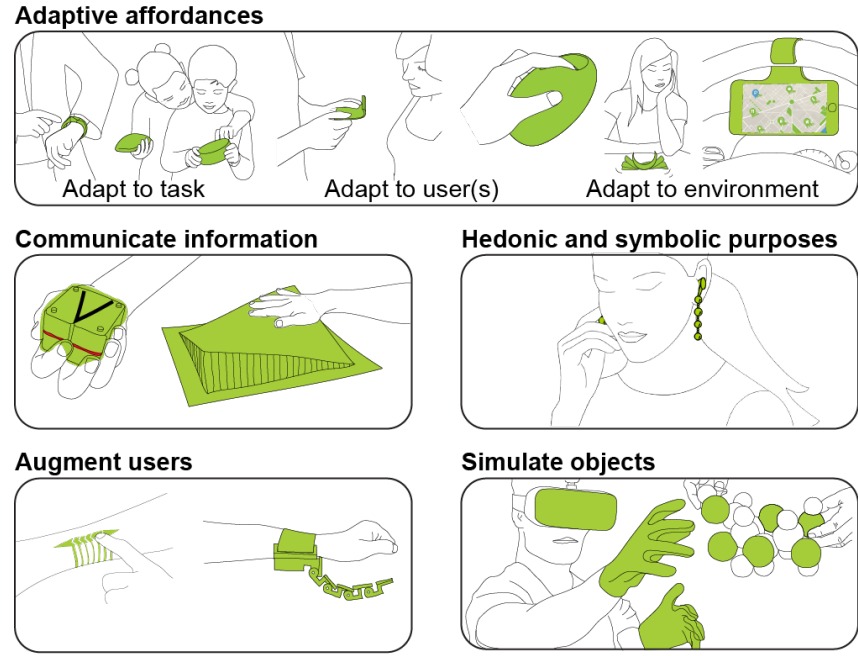

Figure 2. Five purposes of shape changes in end-user interactive devices.

\section{Shape-Changing Interfaces as a Research Field}

Shape-changing interface research has its roots in Tangible User Interfaces [81] and Organic User Interfaces [37] and draws on expertise from a range of disciplines. Current work has many different foci including engineering (e.g. robotics, material engineering), behavioural science, and (interaction) design. Each of these individual fields have goals of their own. For example, haptics researchers strive to generate and 'display' kinaesthetic and tactile stimuli to the user [86]; while robotics researchers seek to build autonomous agents that accomplish given tasks through interactions with the environment [3]. These foci allow researchers to investigate the problem space from several directions, often combining diverse methodological approaches.

However, research into shape-changing interfaces has its own goals and constraints that make it a unique field. These span the technical challenges of complexity and robustness, to users' emotional response, and the device's wider impact on society. Our belief is that the unique challenges, constraints, and the interactive nature of shape-changing interfaces will drive scientific breakthroughs in technology, perception, and HCI that would be fundamentally different than if only approached from one of these other fields. The field of shape-changing interfaces has a great opportunity to bridge between these different disciplines, contributing not only new research, but also to the cross pollination of concepts and techniques.

\section{PURPOSES AND BENEFITS}

To invest resources into addressing the grand challenges outlined in this article, researchers and target user-groups must see clear benefits in the development of shape-changing interfaces. This section briefly explores the purposes and benefits from the literature (summarized in Figure 2). We see 'benefits' as tightly interwoven with the device's (current) intended purpose. The categories are adapted and updated from the purpose categories presented by Rasmussen et al. [72], using the output from a three-day workshop (detailed later). 


\section{Adaptive Affordance}

By adapting its shape to specific contexts of interaction, objects can increase their usefulness and extend their reusability through changing their physical affordances. The literature discusses these changes as dynamic affordance [23, 72], justin-time affordance [9] or dynamic ergonomics [34].

Adaptation to the task involves shape-change to facilitate the execution of an action, such as a mobile phone that mutates into a game controller [76], increasing device reusability. Adaptation to the $u \operatorname{ser}(s)$ involves shape change to facilitate the execution of an action by a specific user(s), for instance, hiding screen content from a passerby [75]. Adaptation to the environment involves shape-changes to support or influence a specific environment; for example, quiet shape-changing message notifications that avoid disrupting a meeting $[14,66]$.

\section{Augment Users}

Augmentation allows the end-user to physically change elements of or within their body. While examples of technologies implanted inside the user's body are rare, we envision cochlear implants becoming interactive to allow adaptation without surgery. External augmentations actuate or become extensions of the user's body. Many examples of external augmentation come from the robotics community (e.g. prosthesis or the sixth finger device [71]) to enhance manipulation dexterity. Conversely, smart fabric can change its stiffness around the user's joints to restrict their movement in gaming, training, or rehabilitation [1].

\section{Simulate Objects}

Shape-changing interfaces have a rich set of applications aimed at simulating static or interactive objects. There are several classes of simulations: (1) Recreating existing real world objects (e.g. car seat adjustment [56] or for remote surgery); (2) Simulating objects that do not yet exist (e.g. in a shape-changing CAD system); (3) Exposing objects that humans could not normally understand (e.g. molecules [11]). Simulating these objects can be achieved through virtual simulation, where users are immersed in virtual content with shape-changing devices providing physical haptic feedback. Physical simulation produces a visual and physical representation of an object that the user can manipulate [58] (e.g. a modular construction kit to represent a beating heart [77]).

\section{Communicate Information}

Shape-changing interfaces encode and communicate information to their users through combinations of visual, haptic (tactile and/or kinaesthetic), and shape animation (transitions between states). A key example of this communication is the new field of data physicalization [47] that extends information visualization into the physical space; for example, by allowing users to physically manipulate a data-set using a dynamic bar chart $[95,96]$. This physicalization allows end-users to exploit their lifelong training in visual and haptic perception.

The haptic element of shape-change can also enable eyes-free interaction - a handheld shape-changing device can inform the user of the directions to take when navigating a map [34, 85] or of incoming messages [14]. Shape may also communicate the state of virtual content [33] in multi-user scenarios.

\section{Hedonic and Symbolic Purposes}

Shape-change can be used for purely hedonic or symbolic purposes - those that have aesthetic, sensorial, or 'fun' goals rather than functional goals [72]. Example hedonic devices include shape-changing garments [7], architecture [63] and works of art [78]. These interfaces are typically used to increase the pleasure or enjoyment of the user(s).

\section{GRAND CHALLENGES}

Our aim is to identify grand challenges in research on shapechanging interfaces. We are inspired by similar attempts in other fields - for example, robotics [24, 109], data physicalization [47], and cross-device interactions [40]. We believe formalising these challenges is timely for this field, whilst acknowledging the discussion around their usefulness [5].

The research challenges in this document were produced over a half-year process. The process was started with a 3-day workshop involving 25 researchers who were experts in shapechanging interfaces, from a variety of disciplines, including design, computer science, human-computer interaction, engineering, robotics, and material sciences. The workshop aimed to discuss the current state-of-the-art in shape-changing interfaces, explore the research challenges of this emerging field, and develop a research agenda for the area. In particular, it included sessions on challenges for the field, benefits of shape-change, and worst-case scenarios. It was synthesized into a preliminary report by the four organizers of the seminar and provided the basis for the current paper. In this way, the procedure is similar to a Delphi study [49] but with more selective reporting. In particular, the challenges presented were regarded of high importance to driving research in this area. We interpret 'high importance' challenges as those that are fundamental to the field's development, and real-world application and deployment. It specifically omits challenges that are common across research areas (for example, the challenges of cross-disciplinary research) and focusses on those that are unique to shape-changing interfaces.

In total, we report 12 grand challenges. The cross-disciplinary nature of this research area is reflected in our categorisation of the challenges into technological, behavioural, design, and societal challenges. Figure 3 shows a summary of the grand challenges we extracted and organized. During the workshop we attempted to prioritise the challenges. However, it quickly became apparent that the strong interconnectivity between the challenges meant it was difficult to isolate and prioritise on "importance"; this article therefore does not offer such a prioritisation. Other classifications, such as on pragmatics of research are of course possible.

\section{TECHNOLOGICAL CHALLENGES}

Developing shape-changing interfaces entails numerous technical challenges on multiple dimensions and in multiple areas. We describe the challenges around toolkits, miniaturisation, integration of shape-change with other I/O modalities, and non-functional requirements.

\section{Toolkits for Prototyping of Shape-changing Hardware}

We see a need to develop toolkits that help prototype the software and hardware components of shape-changing inter- 


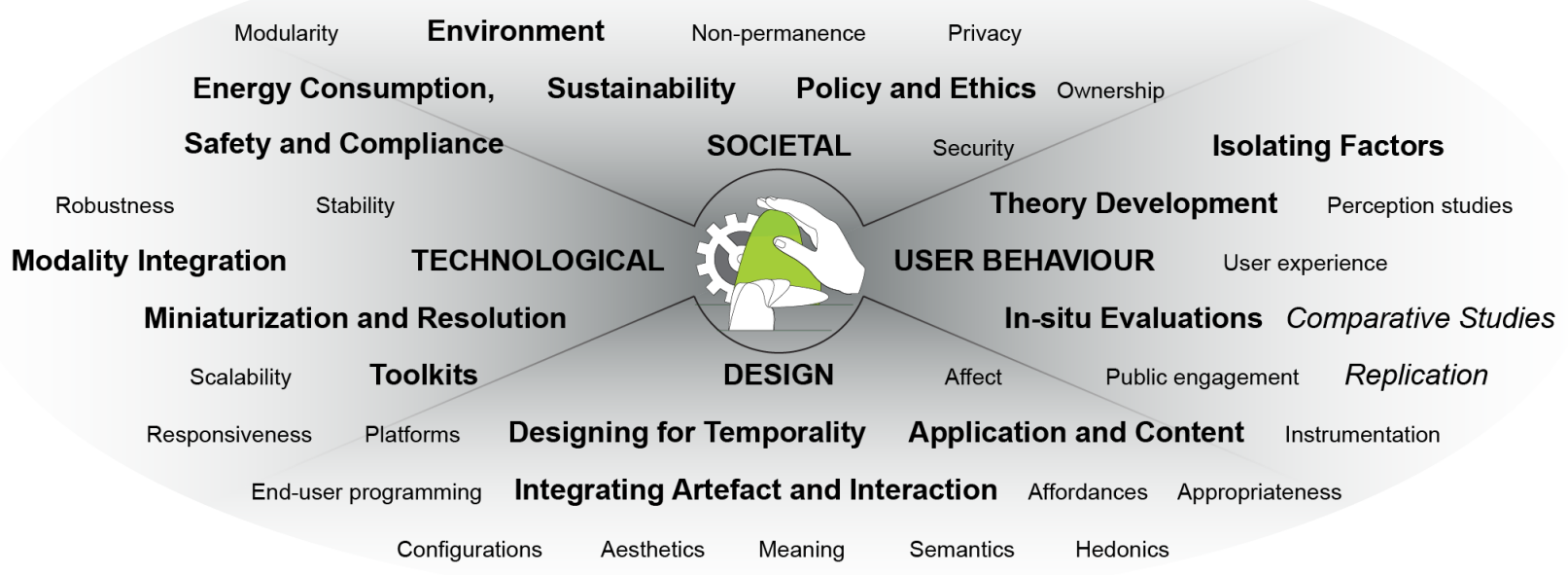

Figure 3. Grand challenges for shape-changing interfaces span technological, behavioural, design, and societal concerns.

faces. This is challenging because prototyping shape-changing interfaces requires knowledge of complex electronics and mechanical engineering that go beyond that typically required in other areas of interactive computing - software programming or simple electronics. This intricate combination of technologies will require further development in physical programming tools [67], rapid prototyping of shape-changing interfaces [20, 32], or technologies such as 4D printing [101]. Furthermore, specific use contexts may require careful selection among actuation approaches [97], that cover electromechanical, pneumatic, or hydraulic actuation, or smart materials [8]. Each of these main classes comprise a multitude of different approaches meaning it is currently excessively difficult to prototype shape-changing interfaces.

We imagine several strands of research to address this challenge: (1) a standard platform for hardware prototyping, dealing with some aspects of actuation; (2) a cross-platform software layer for applications and; (3) tools for end-user programming. Recent research has started to address this challenge at all three layers $[32,64,105]$. The next steps are to better understand requirements from existing prototypes and taxonomy papers before creating a platform that enables the sharing of knowledge and code across the whole community.

The purpose of toolkits is to dramatically lower the barrier to implementation. A suitable goal is to reduce the implementation effort of classic interfaces (e.g. InForm [23] or PinWheels [45]) by at least a factor of 10 in time and cost.

\section{Miniaturized Device Form Factors and High Resolution}

A significant challenge remains in the availability of small form-factor, minimal weight, and high resolution actuators. In line with more general computing trends, shape-changing interfaces are moving from stationary to mobile to wearable form factors [1, 46], and from rigid to flexible to stretchable and even floating shapes [27, 57, 62, 65]. The use of electromechanical actuators, as is common in many systems (e.g. inFORM [23], PinWheels [45], the BMW Kinetic sculpture [90]), often results in large, heavy, and immobile setups that are not compatible with these demands. In addition, enduser expectations of current interactive systems will demand high-resolution output-Humans' haptic and visual perception still far exceeds that possible in shape-changing interfaces [48]. However, increasing the shape resolution [75] tends to considerably complicate the technical setup.

We believe this challenge can be successfully addressed by building on recent results from soft- and modular-robotics, and smart materials. First activities should focus on soft robotics, as this field is the closest to supporting working prototypes. Soft robotics are often based on pneumatic approaches [1, 22, $65,108]$, which allow for varied geometries, slim shapes, and even stretchable form factors, while offering strong output forces. Recent advances in computational design and digital fabrication show great potential in simplifying the implementation of custom form factors and customized actuation patterns. The next important steps consist of increasing the resolution and the actuation speed.

Smart materials can further help to miniaturize form factors to slim films or sheets. Coelho [9] demonstrated the viability of shape memory alloys for use in HCI prototypes, but they have high energy consumption, slow response time, and heat spread. These limitations can be overcome by identifying designs that carefully fine-tune mechanical behaviour [25]. Dielectric elastomers [8] can support more form factors, while offering fast response times. It will be important to identify ways to increase their actuation strengths, for instance by combining multiple layers of elastomers. Lastly, fully printed actuators [82] promise to speed up the fabrication of slim actuators, but at the cost of limited actuation speed and strength.

The modular robotics approach, where interfaces are formed by assemblies of units, provides a promising approach for scalability in shape-change. In current systems, the individual 
units are still at the cm-scale [32, 53, 76, 77]: the key challenge for shape-changing interfaces is to downscale the individual units, such that future interfaces will allow users to interact with "stuff rather than things" [53] and hence support a much higher resolution and expressiveness of shape-change.

A realistic goal is to develop actuation systems that are 12 orders of magnitude slimmer and more lightweight than current systems, while they respond in real time and produce forces comparable to the electromechanical actuators that are used in most current shape-changing interfaces. The output resolution should be in the mm-scale rather than in the $\mathrm{cm}$ scale. Given current progress, modular actuation systems should scale to 100's or even 1000's of units, rather than the 10 's demonstrated today.

\section{Integration of Additional I/O Modalities}

Today's shape-changing interfaces typically focus on shape input and output, with less emphasis on other modalities-for instance, visual output is often realised by projection mapping or low resolution LEDs. While this is acceptable for early research prototypes, successful real-world interfaces will demand additional input and output modalities. This includes high-resolution touch sensing, in-place visual output, adjustable material properties and accurate sensing of the device's physical shape. Without this core integration, shapechanging interfaces will fail to realise their full potential.

We see multiple important directions to address this challenge. First, conventional off-the-shelf sensors and active displays (e.g. LCD, OLED) should be integrated in shape-changing interfaces. Second, advances in flexible sensors and displays open up a path toward integrating conformal touch sensors and displays that are directly integrated with the actuator. For instance, elastic conductors can be printed to capture touch input on deforming surfaces [106], while electroluminescent materials [61] or printed OLEDs actively emit light.

In addition to high-resolution visual output, we believe that future shape-changing interfaces should render haptic material properties, in addition to shape alone. Recent research has provided first evidence of the feasibility of rendering various basic material properties even with a pin-type display [58].

A suitable goal is to realize shape-changing interfaces that capture touch input and provide high-resolution visual output in a quality comparable to today's handheld computing devices, while having the capability to dynamically modify their haptic material properties such that they feel realistic to the user.

\section{Non-functional Requirements}

Energy consumption is a significant challenge in actuated systems, particularly in mobile or wearable solutions which must be self-contained. Today's shape-changing interfaces are usually tethered; if mobile, they typically use large batteries or have short battery life-spans that would be prohibitive in realistic use settings. While there is little room for improving the energy consumption of basic actuation technologies, we see significant potential in systems design that reduces power consumption by offering (bi-)stable states [29, 88]. In these cases, the system only requires energy for transitioning between states, but does not consume any power when residing in a state. Further, there is potential for applying environmental energy harvesting to shape-changing interfaces-ambient light, thermal radiation, and user movement seem promising sources. Notwithstanding these techniques, devices that can self-charge [13] will ensure the burden of charging is removed from end-users. A suitable goal for this challenge is to realize mobile and wearable shape-changing interfaces that can be used for at least a full day without recharging.

Safety and Compliance are also significant challenges to address before placing actuated objects in users' hands. Robotics researchers are now moving beyond traditional robotic arms, wheeled platforms and humanoids to explore a wide variety of different form factors, especially influenced by the goal of bringing robots out of the industrial setting into closer proximity to humans. For example, soft robots made of flexible and elastic silicone, with pneumatic actuation can deform in size while remaining soft and compliant, so can be used close to, or even on people's bodies.

\section{USER BEHAVIOUR CHALLENGES}

This section describes research challenges concerning users' understanding of, interaction with, and behaviour around shape-changing interfaces. Addressing these challenges will produce empirical and theoretical insight about behaviour.

\section{Understanding the User Experience of Shape-change}

We should seek to understand the user experience (UX) of shape-change. Evaluating the user experience and usefulness of new technology is challenging in general, but shape-change poses a specific set of challenges. First, shape-change combines modalities (e.g. visual, haptic) - isolating their relative effects is hard. Second, the experience of using shape-change spans diverse goals such as communicating affect, reducing task completion time, and task fit. Third, current systems are often not robust enough for in-depth evaluations. So despite calls for more studies, this challenge remains open.

By understanding the user experience of shape-changing interfaces we will be able to characterise their value, understand in which domains and for what tasks they are beneficial, and support their design and construction. We see two parts to this challenge: suitable evaluation and the isolation of outcomes.

\section{In-situ, Comparative, and Replicated UX Evaluations}

Current research in shape-changing interfaces is limited by the complexity of hardware (as described earlier) which results in many devices being fragile, hard to replicate, and not suitably robust for long term use. One consequence is that in-situ evaluations of shape-change with real tasks are rare (but do exist $[21,30,66])$. Such evaluations will help to assess suitable contexts of use, the fit between tasks and interfaces, and issues around the cultural appropriation of shape-change.

Further, there is a need for more comparative and controlled studies that allow us to better understand the benefits and drawbacks of shape-changing interfaces compared to existing modailites of interaction, including direct manipulation 
in GUIs, un-actuated TUIs, gestural interaction in spatial virtual reality, and single point haptic feedback. Research in the evaluation of TUIs has proven insightful (e.g. Tuddenham et al. [103] and Zuckerman and Gal-Oz [110]). Comparing physical shape-change to visual-only changes is a first important step, but we need to move beyond this to justify the cost and complexity of shape-changing interfaces.

Finally, replications of work on shape-change are rarely conducted. Most dissemination venues favour novelty and the unique one-off prototypes; the cost of (re-)building previously reported systems prevent independent replication studies (although advances in toolkits would significantly help). The value of replications are well-argued within and outside of HCI $[18,38]$. An important first step is to see if findings obtained with non-functional prototypes (e.g. videos [68]) can be replicated with physical prototypes.

\section{Isolating Factors and Outcomes of Shape-change}

Most evaluations of shape-change concern an entire system. To deepen evaluation we must isolate the factors and outcomes of different aspects of these systems. Key factors to consider are the experience of different transformations among shapes, the impact of using shape-change as it unfolds, and interacting not only above or on shape-changing interfaces, but also within them (through deformation and pressure). The impact of scale and resolution on experience is also a key question: a first step would be just-noticeable-difference studies of motion resolution (compared to Shimojo et al.'s static investigation [83]) and understanding the influence of scale on experience.

Further, there is a need to explore detailed outcomes of using shape-change: learnability and naturalness of interaction are claimed as benefits, but to our knowledge they are not studied so far. Many papers have linked shape-change and the experience of particular feelings $[68,89,98]$. However, several of these papers remain unclear about their basic claims of affect [19]. For instance, are claims being made about core affect (a non-reflective, always available feeling) or emotion (involving cognitive appraisal and attribution)?

Similarly, our field would benefit from concepts identified in Human Robot Interaction (HRI). HRI researchers often investigate human perception of anthropomorphism, animacy, likeability, perceived intelligence, and perceived safety in robots [4] or improved techniques for robots to convey intent through non-verbal motion. An important next step will consist of investigating to what extent these predominantly agent-based findings transfer to shape-changing interfaces.

\section{Shape-Change Theory Building}

Behavioural sciences often construct theories in an attempt to integrate and explain empirical results, to identify gaps in our understanding, and to make predictions. Shape-change's grand challenge is to integrate and explain our empirical results and make predictions about the use of future shape-changing interfaces. We are unaware of theorizing in this sense about shape-change (as opposed to research in tangible user interfaces, where work has begun [39, 43]). The benefits of such work would be to generalize and predict the user experience and usefulness of specific examples of shape-change.
Current use and development of theory in shape-change research is rare. While examples include biological theories of motion [36, 51], most theory use in shape-change has concerned affordance $[23,55,60,100]$. The notion of affordance has helped drive technical development, analyse empirical data, and generate experimental prototypes. It has not yet, to our knowledge, been used to formulate an integrated account of empirical data about shape-change, nor to make predictions. Nor are we aware of the application of theories about graphical perception, biological growth, or movement control- to take a few examples-in shape-change research.

Current research needs integrated accounts of what shapechange is, why it works, what experiences it can engender, and when it is useful. We imagine as a first step that this could use Dubin's [16] view of theory-building. A key part of his work is propositions-statements linking concepts through laws of interactions and boundary conditions. Propositions contain actual, committing statements describing how concepts influence each other. For shape-change research, we need to develop theoretical statements that articulate propositions on how shape-change affects interaction. This would help us to develop scientific claims about shape-change as well as predict how users might react to new shape-changing interfaces.

\section{DESIGN CHALLENGES}

This section describes the design research challenges. The biggest differences to the traditional field of design is the responsiveness of shape-changing interfaces, the design and form of the artefacts, and their dynamic qualities. Shapechanging interfaces are unique in the integration of movement and transformation through actuation based on direct user input. Three key design challenges are identified: designing for temporality, integrating the artefact and interaction, and developing applications and content.

\section{Designing for Temporality}

Shape-changing interfaces require temporal design: there is an important challenge in translating behavioural sketches and functional descriptions of behaviour into actual designs. While sketches and prototypes of static forms provide representations that are visually and tangibly comparable by multiple people simultaneously, dynamic form has temporal aspects that are difficult to compare in parallel. This makes traditional methods such as design critique [54] more difficult to perform. In particular, the direct interaction a user has with a shape-changing interface offers a unique experience to that person; there is not yet a language that supports the articulation of properties, experiences, or sensations, such as colour or material properties in traditional product design.

The many disciplines of design have started the first steps in addressing this challenge. Dance and movement [41] has inspired work into active form [73], dynamic form [74], and temporal form [104]. Designers use 'acting out' and choreography to sketch dynamic form languages and implement them into experiential prototypes [42, 74], building on the body of knowledge available in animation (e.g. in the computational design of mechanical characters [12]). Inspiration may also be drawn from music, where notation systems use 
symbols to indicate music both concretely (notes) and more ambiguously through expressions such as "crescendo poco a poco' [35]. Other directions can be found in modelling platforms with multiple degrees of freedom that replicate different movement patterns in parallel, although interaction will again lead to diverse experiences [52]. A key goal is the development of techniques and methodologies that allow the design, construction, and direct comparison of temporal forms.

\section{Integrating Artefact and Interaction}

With shape-changing interfaces, designers will be challenged to develop devices that are satisfying both in the form and dynamics of interaction between the artefact and the people interacting with it [74]. The usability and aesthetics will be inextricably linked, with the aim of designing shape-changing materials that engage the body as well as our mind [69].

This interplay of properties increases the complexity of the design exponentially, and consequently the training required by designers. Their expanding duties that were previously separated - the notion of a "master builder" [50] in architecture-reflects the increasing responsibility and purview of the designer. As part of this, designers need to understand theory, heuristics, and dynamic affordances where appearance and actions serve as carriers of meaning [15]. Expanding their competences is therefore essential for the future development of this technology. A first step is to develop more tools that support the design process (as per the technology challenges) and design methods that couple action and reaction [87]. A suitable measure of success is the development of design tools and methods that intrinsically integrate artefact and interaction in the design process; and designers whose training means they can successfully handle this complexity.

\section{Application and Content Design}

There is a significant challenge in the 'when', 'what', and 'how' of the design and implementation of applications and content. We can break this challenge into four parts: (1) When should we apply shape-change? (2) What shape-changes should we apply? (3) What applications should we build? (4) How do we design the content for those applications?

When to apply shape-change: While shape-changing interfaces provide many advantages, they are clearly not suitable for all interaction scenarios. Possibly due to the lack of theoretical understanding, the community has yet to explore or understand under which circumstances physically-dynamic interfaces are appropriate and when traditional interfaces should be used. However, first broad steps are underway, with researchers presenting exemplars at large public events [44], sharing prototypes with the broader public [93], and developing speculative scenarios and designs [17, 92] -all of which trigger discussions on acceptance and desirability. A obtainable goal is to develop frameworks and design principles that describe when shape-change should be applied and when traditional interfaces are more appropriate.

What shape-changes to apply: One of the key benefits of shape-changing interfaces is their ability to transform into multiple shapes or forms, potentially for quite diverse tasks or situations. Consequently, the designer can choose shapes that are adapted to the human instead of technical, manufacturing, or transportation limitations. However, there is a lack of understanding as to what types of change should occur in more abstract or generic circumstances, where shape may not naturally represent content. Without clear guidelines of use, there is the risk that end-users will become confused by semantics across different shape-change applications. Using design guidelines, researchers should aim to reach a consensus on shape-change semantics that can apply across many devices.

Applications: The literature contains a vast range of specific shape-changing interface prototypes (see Figure 2) with several initial attempts made to identify categories of applications [21, 93]. This diverse set of applications allows researchers to explore and test the design space; but discovering the 'killer-apps' and domains for shape-changing interfaces is what will dictate its ultimate success-without this, the technology may never make it into consumer's hands. Researchers, in conjunction with industrialists, should identify and explore key application domains that provide clear benefits and routes to end-user engagement. Without more targeted application domains (such as Data Physicalization [47]), the field runs the risk of becoming too diverse and being unable to cohesively tackle many of the grand challenges described here.

Content design: All shape-changing interfaces will require careful consideration of the end-user content. This is particularly relevant for shape-changing interfaces that exploit the dominant visual sense through a shape-changing display. Such content must address the visual and physical configuration of the display and user input. First steps have already begun to address non-traditional display formats (e.g. spherical, volumetric displays, and 3D UI's [6] and non-rectangular displays $[79,80])$. Research must then address content design for displays that change their shape. Researchers should aim to develop toolkits that facilitate content design on any size, shape, or form-factor interface.

\section{POLICY, ETHICS, AND SUSTAINABILITY CHALLENGES}

The regulatory, ethical and sustainability implications of the development of shape-changing interfaces will impact the long-term adoption of this technology, therefore also its longterm development.

\section{Policy and Ethics}

We see a significant challenge for policy-makers to create legislation that ensures the safe and ethical operation of shapechanging interfaces. It is particularly challenging to ensure that these interfaces are suitably regulated without curtailing their innovation potential, especially with the large diversity of devices that may become available.

Shape-changing interfaces pose a number of safety and ethical risks that without suitable regulation may result in rejection by end-users or widespread bans by government. The key issues for shape-changing interfaces are: (1) Safety: they must be built so as not to harm users or bystanders or cause damage to physical property; (2) Security, privacy, and control: they must be subject to the same security and privacy expectations of other digital systems (both digitally and physically). Endusers must ultimately always have control over the forms their 
interface may take; (3) Content and appropriate use: they must prevent inappropriate content being shown to particular user groups (e.g. children). Legislation should also provide guidance on inappropriate use cases based on societal, cultural, and criminal values; (4) Ownership and responsibility: individual users are likely to own the hardware for these devices (just as we own mobile phones today) with remote service providers delivering content. The law must clearly distinguish the responsibility of different parties for the output and its consequences; (5) Implications of non-permanency: today's shape-changing objects do so in a predictable and repeatable fashion. The radical transformations proposed for the future may impact on areas of society that require a known 'history' of form; e.g. the untraceable transformation of a weapon used in an attack could hinder crime-solving teams.

Governments often struggle to legislate on new technological advances quickly enough. However, our field can learn lessons from other step-change technologies (e.g. drones, autonomous cars) on how to rapidly and suitable legislate to avoid stifling innovation. To the best of our knowledge, no such explorations exist for shape-changing interfaces.

\section{Sustainability and the Environment}

Shape-changing interfaces are a 'double-edged sword' for sustainability. The combination of physical actuation components, display surfaces, and electronics, pose an increased demand on natural resources, and increased challenges for recycling, and later re-use beyond those already present in consumer-level electronics [59]. Addressing these resource challenges are essential for the long-term viability of shape-changing interfaces and the health of our environment.

However, a shape-changing interface's morphing ability should ultimately reduce the need for multiple instances of similar devices and therefore reduce long-term resource requirements. For example, instead of consumers needing an array of different form-factor computing devices (laptop, tablet, smartphone, smartwatch), a single shape-changing device could transform into the desired size, shape, and weight on demand.

To reach such a state, researchers should place emphasis on developing 'life-time' shape-changing interfaces: those that have the life-span of decades (similar to a house) rather than the few years typical in today's consumer electronics [2][107, pg. 258]. To achieve this, researchers must strive for modularity [31] to allow the repair, self-repair [99], or replacement of broken components, and support upgrades as higher 'shape resolution' [75] components are developed. Standardization that can survive the fundamental redesign of these interfaces will be essential. Finally, by supporting interoperability between software and hardware, developers will further reduce the burden on the resources necessary to produce these devices.

\section{CONCLUDING REMARKS}

The field of shape-changing interfaces has greatly expanded during the last few years, showcasing novel technologies and embracing new disciplines. At the same time, the field has matured considerably, and is beginning to converge on key questions surrounding technology, design, theory-building, and societal implications. In this paper we have identified and discussed what we consider the 12 grand challenges currently facing researchers. Although there are many examples of shape-changing interfaces that provide delightful experiences and offer better ergonomics, the challenges identified need be addressed to help deliver the full benefits of shape-change.

It is important that we also recognize and deal with limitations of shape-changing interfaces and temper this optimism. Shneiderman [84] discussed the ten plagues of the information age, many of which apply directly to shape-changing research. In research on proxemics, researchers have discussed sinister "dark patterns" [28], that is, applications of proxemics where users are manipulated and deceived. We appreciate the transparency and open dialogue encouraged by such work, which highlights how things can go wrong. We envision similar negative and even dangerous appropriations of shape-changing interfaces will also occur; understanding and minimising that impact crosses many of the presented challenges.

We are excited about the potential of shape-changing interfaces and how they will transform interaction with computing systems. We see the identification of these grand challenges as a positive step in focussing research efforts towards this common goal. These challenges will, no doubt, evolve as technology matures, society changes, and our understanding of users increases. We hope you will join us in developing these interfaces to shape the future of human-computer interaction.

\section{ACKNOWLEDGEMENTS}

This work originated from the discussions held at Dagstuhl Seminar \#17082 on Shape-Changing Interfaces; we'd like to acknowledge and thank all attendees at this workshop for their input. The authors in this paper are partially supported by grants from: MORPHED (EPSRC \#EP/M016528/1), GHOST (EC FET-open \#309191), Automorph (EPSRC \#EP/P004342/1), The Leverhulme Trust, InteractiveSkin (ERC Starting Grant \#714797), and Body-UI (ERC Consolidator Grant \#648785).

\section{REFERENCES}

1. Ahmed Al Maimani and Anne Roudaut. 2017. Frozen Suit: Designing a Changeable Stiffness Suit and Its Application to Haptic Games. In Proceedings of the 2017 CHI Conference on Human Factors in Computing Systems (CHI '17). ACM, New York, NY, USA, 2440-2448. DOI :

http://dx.doi.org/10.1145/3025453.3025655

2. Anders S. G. Andrae and Otto Andersen. 2010. Life Cycle Assessments of Consumer Electronics-Are they Consistent? The International Journal of Life Cycle Assessment 15, 8 (01 Sep 2010), 827-836. DOI: http://dx.doi .org/10.1007/s11367-010-0206-1

3. Minoru Asada. 1995. Preface. Advanced Robotics 10, 2 (1995), 139-142. DOI: http://dx.doi.org/10.1163/156855396X00273

4. Christoph Bartneck, Dana Kulić, Elizabeth Croft, and Susana Zoghbi. 2009. Measurement Instruments for the Anthropomorphism, Animacy, Likeability, Perceived Intelligence, and Perceived Safety of Robots. 
International Journal of Social Robotics 1, 1 (01 Jan 2009), 71-81. DOI :

http://dx.doi.org/10.1007/s12369-008-0001-3

5. Jordan Beck and Erik Stolterman. 2017. Reviewing the Big Questions Literature;: Or, Should HCI Have Big Questions?. In Proceedings of the 2017 Conference on Designing Interactive Systems (DIS '17). ACM, New York, NY, USA, 969-981. DOI :

http://dx.doi.org/10.1145/3064663.3064673

6. Hrvoje Benko, Andrew D. Wilson, and Ravin Balakrishnan. 2008. Sphere: Multi-touch Interactions on a Spherical Display. In Proceedings of the 21st Annual ACM Symposium on User Interface Software and Technology (UIST '08). ACM, New York, NY, USA, 77-86. DOI : http://dx. doi .org/10.1145/1449715.1449729

7. Joanna Berzowska and Marcelo Coelho. 2005. Kukkia and Vilkas: Kinetic Electronic Garments. In Ninth IEEE International Symposium on Wearable Computers (ISWC'05). 82-85. D0I :

http://dx.doi.org/10.1109/ISWC.2005.29

8. Paul Brochu and Qibing Pei. 2010. Advances in Dielectric Elastomers for Actuators and Artificial Muscles. Macromolecular rapid communications 31, 1 (2010), 10-36.

9. Marcelo Coelho, Hiroshi Ishii, and Pattie Maes. 2008. Surflex: A Programmable Surface for the Design of Tangible Interfaces. In $\mathrm{CHI}$ '08 Extended Abstracts on Human Factors in Computing Systems (CHI EA '08). ACM, New York, NY, USA, 3429-3434. DOI : http://dx.doi.org/10.1145/1358628.1358869

10. Marcelo Coelho and Jamie Zigelbaum. 2011. Shape-changing Interfaces. Personal and Ubiquitous Computing 15, 2 (01 Feb 2011), 161-173. DOI : http://dx.doi.org/10.1007/s00779-010-0311-y

11. Daniel M Cohen and Christopher S Chen. 2008. Mechanical Control of Stem Cell Differentiation. StemBook [Internet] (October 2008). DOI : http://dx.doi.org/10.3824/stembook.1.26.1

12. Stelian Coros, Bernhard Thomaszewski, Gioacchino Noris, Shinjiro Sueda, Moira Forberg, Robert W. Sumner, Wojciech Matusik, and Bernd Bickel. 2013. Computational Design of Mechanical Characters. ACM Trans. Graph. 32, 4, Article 83 (July 2013), 12 pages. DOI : http://dx.doi.org/10.1145/2461912.2461953

13. Artem Dementyev, Hsin-Liu (Cindy) Kao, Inrak Choi, Deborah Ajilo, Maggie Xu, Joseph A. Paradiso, Chris Schmandt, and Sean Follmer. 2016. Rovables: Miniature On-Body Robots As Mobile Wearables. In Proceedings of the 29th Annual Symposium on User Interface Software and Technology (UIST '16). ACM, New York, NY, USA, 111-120. DOI : http://dx.doi.org/10.1145/2984511.2984531

14. Panteleimon Dimitriadis and Jason Alexander. 2014. Evaluating the Effectiveness of Physical Shape-change for In-pocket Mobile Device Notifications. In
Proceedings of the SIGCHI Conference on Human Factors in Computing Systems (CHI'14). ACM, New York, NY, USA, 2589-2592. DOI :

http://dx.doi.org/10.1145/2556288.2557164

15. Tom Djajadiningrat, Stephan Wensveen, Joep Frens, and Kees Overbeeke. 2004. Tangible Products: Redressing the Balance between Appearance and Action. Personal and Ubiquitous Computing 8, 5 (01 Sep 2004), 294-309. DOI : http://dx.doi .org/10.1007/s00779-004-0293-8

16. Robert Dubin. 1969. Theory Building. Free Press.

17. Anthony Dunne and Fiona Raby. 2013. Speculative Everything: Design, Fiction, and Social Dreaming. MIT Press.

18. Nature Editorial. 2016. Go forth and replicate! 536, 7617 (August 2016), 373. DOI :

http://dx.doi.org/10.1038/536373a

19. Panteleimon Ekkekakis. 2013. The Measurement of Affect, Mood, and Emotion: A Guide for Health-behavioral Research. Cambridge University Press.

20. Aluna Everitt and Jason Alexander. 2017. PolySurface: A Design Approach for Rapid Prototyping of

Shape-Changing Displays Using Semi-Solid Surfaces. In Proceedings of the 2017 Conference on Designing Interactive Systems (DIS '17). ACM, New York, NY, USA, 1283-1294. DOI :

http://dx.doi.org/10.1145/3064663.3064677

21. Aluna Everitt, Faisal Taher, and Jason Alexander. 2016. ShapeCanvas: An Exploration of Shape-Changing Content Generation by Members of the Public. In Proceedings of the 2016 CHI Conference on Human Factors in Computing Systems (CHI'16). ACM, New York, NY, USA, 2778-2782. DOI :

http://dx.doi.org/10.1145/2858036.2858316

22. Sean Follmer, Daniel Leithinger, Alex Olwal, Nadia Cheng, and Hiroshi Ishii. 2012. Jamming User Interfaces: Programmable Particle Stiffness and Sensing for Malleable and Shape-changing Devices. In Proceedings of the 25th Annual ACM Symposium on User Interface Software and Technology (UIST'12). ACM, New York, NY, USA, 519-528. DOI :

http://dx.doi.org/10.1145/2380116.2380181

23. Sean Follmer, Daniel Leithinger, Alex Olwal, Akimitsu Hogge, and Hiroshi Ishii. 2013. inFORM: Dynamic Physical Affordances and Constraints Through Shape and Object Actuation. In Proceedings of the 26th Annual ACM Symposium on User Interface Software and Technology (UIST'13). ACM, New York, NY, USA, 417-426. DOI :

http://dx.doi.org/10.1145/2501988.2502032

24. SPARC The Robotics Partnership for Europe. 2016. Robotics 2020 Multi-Annual Roadmap. Technical Report. Retrieved 15/09/17 from https://www.eu-robotics.net/cms/upload/topic_groups/ H2020_Robotics_Multi-Annual_Roadmap_ICT-2017B.pdf 
25. Stephen J Furst, George Bunget, and Stefan Seelecke. 2013. Design and Fabrication of a Bat-inspired Flapping-flight Platform using Shape Memory Alloy Muscles and Joints. Smart Materials and Structures 22, 1 (2013). DOI : http://dx.doi.org/10.1088/0964-1726/22/1/014011

26. Antonio Gomes, Andrea Nesbitt, and Roel Vertegaal. 2013. MorePhone: A Study of Actuated Shape Deformations for Flexible Thin-film Smartphone Notifications. In Proceedings of the SIGCHI Conference on Human Factors in Computing Systems (CHI '13). ACM, New York, NY, USA, 583-592. DOI : http://dx.doi.org/10.1145/2470654.2470737

27. Antonio Gomes, Calvin Rubens, Sean Braley, and Roel Vertegaal. 2016. BitDrones: Towards Using 3D Nanocopter Displays As Interactive Self-Levitating Programmable Matter. In Proceedings of the 2016 CHI Conference on Human Factors in Computing Systems (CHI '16). ACM, New York, NY, USA, 770-780. DOI : http://dx.doi.org/10.1145/2858036.2858519

28. Saul Greenberg, Sebastian Boring, Jo Vermeulen, and Jakub Dostal. 2014. Dark Patterns in Proxemic Interactions: A Critical Perspective. In Proceedings of the 2014 Conference on Designing Interactive Systems (DIS '14). ACM, New York, NY, USA, 523-532. DOI : http://dx.doi.org/10.1145/2598510.2598541

29. Daniel Groeger, Elena Chong Loo, and Jürgen Steimle. 2016. HotFlex: Post-print Customization of 3D Prints Using Embedded State Change. In Proceedings of the 2016 CHI Conference on Human Factors in Computing Systems (CHI '16). ACM, New York, NY, USA, 420-432. DOI : ht tp://dx.doi.org/10.1145/2858036.2858191

30. Erik Grönvall, Sofie Kinch, Marianne Graves Petersen, and Majken K. Rasmussen. 2014. Causing Commotion with a Shape-changing Bench: Experiencing Shape-changing Interfaces in Use. In Proceedings of the SIGCHI Conference on Human Factors in Computing Systems (CHI '14). ACM, New York, NY, USA, 2559-2568. DOI : http://dx.doi.org/10.1145/2556288.2557360

31. Dave Hakkens. 2016. Phoneblocks. (2016). Retrieved 15/09/17 from www. phonebloks. com.

32. John Hardy, Christian Weichel, Faisal Taher, John Vidler, and Jason Alexander. 2015. ShapeClip: Towards Rapid Prototyping with Shape-Changing Displays for Designers. In Proceedings of the 33rd Annual ACM Conference on Human Factors in Computing Systems (CHI '15). ACM, New York, NY, USA, 19-28. DOI : http://dx.doi.org/10.1145/2702123.2702599

33. Nikolaj Haulrik, Rasmus M. Petersen, and Timothy Merritt. 2017. CADLens: Haptic Feedback for Navigating in 3D Environments. In Proceedings of the 2017 ACM Conference Companion Publication on Designing Interactive Systems (DIS '17 Companion). ACM, New York, NY, USA, 127-131. DOI : http://dx.doi.org/10.1145/3064857.3079132
34. Fabian Hemmert, Susann Hamann, Matthias Löwe, Josefine Zeipelt, and Gesche Joost. 2010. Shape-changing Mobiles: Tapering in Two-dimensional Deformational Displays in Mobile Phones. In CHI '10 Extended Abstracts on Human Factors in Computing Systems (CHI EA '10). ACM, New York, NY, USA, 3075-3080. DOI : http://dx.doi.org/10.1145/1753846.1753920

35. Bart Hengeveld. 2015. Composing Interaction: Exploring Tangible Notation Systems For Design. In Proceedings of the Ninth International Conference on Tangible,

Embedded, and Embodied Interaction (TEI '15). ACM, New York, NY, USA, 667-672. DOI :

http://dx.doi.org/10.1145/2677199.2687915

36. Guy Hoffman and Wendy Ju. 2014. Designing Robots with Movement in Mind. Journal of Human-Robot Interaction 3, 1 (2014), 89-122. DOI: http://dx.doi.org/10.5898/JHRI.3.1.Hoffman

37. David Holman and Roel Vertegaal. 2008. Organic User Interfaces: Designing Computers in Any Way, Shape, or Form. Commun. ACM 51, 6 (June 2008), 48-55. DOI : http://dx.doi.org/10.1145/1349026.1349037

38. Kasper Hornbæk, Søren S. Sander, Javier Andrés Bargas-Avila, and Jakob Grue Simonsen. 2014. Is Once Enough?: On the Extent and Content of Replications in Human-computer Interaction. In Proceedings of the SIGCHI Conference on Human Factors in Computing Systems (CHI '14). ACM, New York, NY, USA, 3523-3532. DOI : http://dx.doi.org/10.1145/2556288.2557004

39. Eva Hornecker and Jacob Buur. 2006. Getting a Grip on Tangible Interaction: A Framework on Physical Space and Social Interaction. In Proceedings of the SIGCHI Conference on Human Factors in Computing Systems (CHI '06). ACM, New York, NY, USA, 437-446. DOI : http://dx.doi.org/10.1145/1124772.1124838

40. Steven Houben, Nicolai Marquardt, Jo Vermeulen, Clemens Klokmose, Johannes Schöning, Harald Reiterer, and Christian Holz. 2017. Opportunities and Challenges for Cross-device Interactions in the Wild. Interactions 24, 5 (Aug. 2017), 58-63. DOI :

http://dx.doi.org/10.1145/3121348

41. Caroline Hummels, Kees C. J. Overbeeke, and Sietske Klooster. 2007. Move to Get Moved: A Search for Methods, Tools and Knowledge to Design for Expressive and Rich Movement-based Interaction. Personal and Ubiquitous Computing 11, 8 (01 Dec 2007), 677-690. DOI: http://dx.doi.org/10.1007/s00779-006-0135-y

42. Yeup Hur, Miriam Sturdee, Migeul Bruns Alonso, Panos Markopoulos, and Jason Alexander. 2017. Fiction and Physicality: A Designerly Approach Towards Complexities of Emerging Technologies. The Design Journal 20, sup1 (2017), 3849-3862. DOI: http://dx.doi.org/10.1080/14606925.2017.1352888 
43. Hiroshi Ishii, Dávid Lakatos, Leonardo Bonanni, and Jean-Baptiste Labrune. 2012. Radical Atoms: Beyond Tangible Bits, Toward Transformable Materials. interactions 19, 1 (Jan. 2012), 38-51. DOI : http://dx.doi.org/10.1145/2065327.2065337

44. Hiroshi Ishii, Daniel Leithinger, Sean Follmer, Amit Zoran, Philipp Schoessler, and Jared Counts. 2015. TRANSFORM: Embodiment of "Radical Atoms" at Milano Design Week. In Proceedings of the 33rd Annual ACM Conference Extended Abstracts on Human Factors in Computing Systems (CHI EA '15). ACM, New York, NY, USA, 687-694. DOI : http://dx.doi.org/10.1145/2702613.2702969

45. Hiroshi Ishii, Sandia Ren, and Phil Frei. 2001. Pinwheels: Visualizing Information Flow in an Architectural Space. In CHI 'O1 Extended Abstracts on Human Factors in Computing Systems (CHI EA '01). ACM, New York, NY, USA, 111-112. DOI : http://dx.doi.org/10.1145/634067.634135

46. Sungjune Jang, Lawrence H. Kim, Kesler Tanner, Hiroshi Ishii, and Sean Follmer. 2016. Haptic Edge Display for Mobile Tactile Interaction. In Proceedings of the 2016 CHI Conference on Human Factors in Computing Systems (CHI '16). ACM, New York, NY, USA, 3706-3716. DOI : http://dx.doi.org/10.1145/2858036.2858264

47. Yvonne Jansen, Pierre Dragicevic, Petra Isenberg, Jason Alexander, Abhijit Karnik, Johan Kildal, Sriram Subramanian, and Kasper Hornbæk. 2015. Opportunities and Challenges for Data Physicalization. In Proceedings of the 33rd Annual ACM Conference on Human Factors in Computing Systems (CHI'15). ACM, New York, NY, USA, 3227-3236. DOI :

http://dx.doi.org/10.1145/2702123.2702180

48. Lynette A. Jones and Susan J. Lederman. 2006. Human Hand Function. Oxford University Press. 52-56 pages.

49. William Jones, Robert Capra, Anne Diekema, Jaime Teevan, Manuel Pérez-Quiñones, Jesse David Dinneen, and Bradley Hemminger. 2015. "For Telling" the Present: Using the Delphi Method to Understand Personal Information Management Practices. In Proceedings of the 33rd Annual ACM Conference on Human Factors in Computing Systems (CHI'15). ACM, New York, NY, USA, 3513-3522. DOI : http://dx.doi.org/10.1145/2702123.2702523

50. Branko Kolarevic. 2004. Architecture in the Digital Age: Design and Manufacturing. Taylor \& Francis.

51. Aleksandra Kupferberg, Stefan Glasauer, Markus Huber, Markus Rickert, Alois Knoll, and Thomas Brandt. 2011. Biological Movement Increases Acceptance of Humanoid Robots as Human Partners in Motor Interaction. AI \& SOCIETY 26, 4 (01 Nov 2011), 339-345. DOI : http://dx.doi.org/10.1007/s00146-010-0314-2

52. Matthijs Kwak and Joep W. Frens. 2015. From Movement to Mechanism: Exploring Expressive
Movement Qualities in Shape-change. In Proceedings of the Ninth International Conference on Tangible, Embedded, and Embodied Interaction (TEI '15). ACM, New York, NY, USA, 461-464. DOI :

http://dx.doi.org/10.1145/2677199.2683580

53. Mathieu Le Goc, Lawrence H. Kim, Ali Parsaei, Jean-Daniel Fekete, Pierre Dragicevic, and Sean Follmer. 2016. Zooids: Building Blocks for Swarm User Interfaces. In Proceedings of the 29th Annual Symposium on User Interface Software and Technology (UIST'16).

ACM, New York, NY, USA, 97-109. D0I : http://dx.doi.org/10.1145/2984511.2984547

54. Kurt Luther, Jari-Lee Tolentino, Wei Wu, Amy Pavel, Brian P. Bailey, Maneesh Agrawala, Björn Hartmann, and Steven P. Dow. 2015. Structuring, Aggregating, and Evaluating Crowdsourced Design Critique. In Proceedings of the 18th ACM Conference on Computer Supported Cooperative Work \&\#38; Social Computing (CSCW'15). ACM, New York, NY, USA, 473-485. DOI : http://dx.doi.org/10.1145/2675133.2675283

55. Timothy Robert Merritt, Mie Nørgaard, Christian $\varnothing$. Laursen, Majken Kirkegård Rasmussen, and Marianne Graves Petersen. 2015. Imagined Physics: Exploring Examples of Shape-changing Interfaces. In Cognitive Robotics. CRC Press LLC, 89-111.

56. G. Michelitsch, J. Williams, M. Osen, B. Jimenez, and S. Rapp. 2004. Haptic Chameleon: A New Concept of Shape-changing User Interface Controls with Force Feedback. In CHI '04 Extended Abstracts on Human Factors in Computing Systems (CHI EA '04). ACM, New York, NY, USA, 1305-1308. DOI :

http://dx.doi.org/10.1145/985921.986050

57. Ken Nakagaki, Artem Dementyev, Sean Follmer, Joseph A. Paradiso, and Hiroshi Ishii. 2016a. ChainFORM: A Linear Integrated Modular Hardware System for Shape Changing Interfaces. In Proceedings of the 29th Annual Symposium on User Interface Software and Technology (UIST '16). ACM, New York, NY, USA, 87-96. DOI : http://dx.doi .org/10.1145/2984511.2984587

58. Ken Nakagaki, Luke Vink, Jared Counts, Daniel Windham, Daniel Leithinger, Sean Follmer, and Hiroshi Ishii. 2016b. Materiable: Rendering Dynamic Material Properties in Response to Direct Physical Touch with Shape Changing Interfaces. In Proceedings of the 2016 CHI Conference on Human Factors in Computing Systems (CHI '16). ACM, New York, NY, USA, 2764-2772. DOI : http://dx.doi.org/10.1145/2858036.2858104

59. I.C. Nnorom and O. Osibanjo. 2008. Overview of Electronic Waste (e-waste) Management Practices and Legislations, and their Poor Applications in the Developing Countries. Resources, Conservation and Recycling 52, 6 (2008), 843-858. D0I : http://dx.doi.org/10.1016/j.resconrec.2008.01.004 
60. Mie Nørgaard, Tim Merritt, Majken Kirkegaard Rasmussen, and Marianne Graves Petersen. 2013. Exploring the Design Space of Shape-changing Objects: Imagined Physics. In Proceedings of the 6th International Conference on Designing Pleasurable Products and Interfaces (DPPI'13). ACM, New York, NY, USA, 251-260. DOI :

http://dx.doi.org/10.1145/2513506.2513533

61. Simon Olberding, Michael Wessely, and Jürgen Steimle. 2014. PrintScreen: Fabricating Highly Customizable Thin-film Touch-displays. In Proceedings of the 27th Annual ACM Symposium on User Interface Software and Technology (UIST'14). ACM, New York, NY, USA, 281-290. DOI :

http://dx.doi.org/10.1145/2642918.2647413

62. Themis Omirou, Asier Marzo, Sue Ann Seah, and Sriram Subramanian. 2015. LeviPath: Modular Acoustic Levitation for 3D Path Visualisations. In Proceedings of the 33rd Annual ACM Conference on Human Factors in Computing Systems (CHI'15). ACM, New York, NY, USA, 309-312. DOI : http://dx.doi.org/10.1145/2702123.2702333

63. Kas Oosterhuis and Nimish Biloria. 2008. Interactions with Proactive Architectural Spaces: The Muscle Projects. Commun. ACM 51, 6 (2008), 70-78.

64. Jifei Ou, Felix Heibeck, and Hiroshi Ishii. 2017. Pneuduino. (2017). Retrieved 15/09/17 from www.pneuduino.org.

65. Jifei Ou, Mélina Skouras, Nikolaos Vlavianos, Felix Heibeck, Chin-Yi Cheng, Jannik Peters, and Hiroshi Ishii. 2016. aeroMorph - Heat-sealing Inflatable Shape-change Materials for Interaction Design. In Proceedings of the 29th Annual Symposium on User Interface Software and Technology (UIST'16). ACM, New York, NY, USA, 121-132. DOI :

http://dx.doi.org/10.1145/2984511.2984520

66. Young-Woo Park, Joohee Park, and Tek-Jin Nam. 2015. The Trial of Bendi in a Coffeehouse: Use of a Shape-Changing Device for a Tactile-Visual Phone Conversation. In Proceedings of the 33rd Annual ACM Conference on Human Factors in Computing Systems (CHI '15). ACM, New York, NY, USA, 2181-2190. DOI : http://dx.doi.org/10.1145/2702123.2702326

67. Amanda Parkes and Hiroshi Ishii. 2010. Bosu: A Physical Programmable Design Tool for Transformability with Soft Mechanics. In Proceedings of the 8th ACM Conference on Designing Interactive Systems (DIS '10). ACM, New York, NY, USA, 189-198. DOI : http://dx.doi.org/10.1145/1858171.1858205

68. Esben W. Pedersen, Sriram Subramanian, and Kasper Hornbæk. 2014. Is My Phone Alive?: A Large-scale Study of Shape Change in Handheld Devices Using Videos. In Proceedings of the 32Nd Annual ACM Conference on Human Factors in Computing Systems (CHI '14). ACM, New York, NY, USA, 2579-2588. DOI : http://dx.doi.org/10.1145/2556288.2557018
69. Marianne Graves Petersen, Ole Sejer Iversen, Peter Gall Krogh, and Martin Ludvigsen. 2004. Aesthetic Interaction: A Pragmatist's Aesthetics of Interactive Systems. In Proceedings of the 5th Conference on Designing Interactive Systems: Processes, Practices, Methods, and Techniques (DIS '04). ACM, New York, NY, USA, 269-276. DOI :

http://dx.doi.org/10.1145/1013115.1013153

70. Ivan Poupyrev, Tatsushi Nashida, and Makoto Okabe. 2007. Actuation and Tangible User Interfaces: The Vaucanson Duck, Robots, and Shape Displays. In Proceedings of the 1st International Conference on Tangible and Embedded Interaction (TEI '07). ACM, New York, NY, USA, 205-212. DOI :

http://dx.doi.org/10.1145/1226969.1227012

71. Domenico Prattichizzo, Monica Malvezzi, Irfan Hussain, and Gionata Salvietti. 2014. The Sixth-Finger: A Modular Extra-finger to Enhance Human Hand Capabilities. In The 23rd IEEE International Symposium on Robot and Human Interactive Communication. 993-998. DOI :

http://dx.doi.org/10.1109/ROMAN.2014.6926382

72. Majken K. Rasmussen, Esben W. Pedersen, Marianne G. Petersen, and Kasper Hornbæk. 2012. Shape-changing Interfaces: A Review of the Design Space and Open Research Questions. In Proceedings of the SIGCHI Conference on Human Factors in Computing Systems (CHI '12). ACM, New York, NY, USA, 735-744. DOI : http://dx.doi.org/10.1145/2207676.2207781

73. Matthias Rauterberg, Ben Salem, and Dirk Van De Mortel. 2005. From Passive to Active Forms. Design and Semantics of Form and Movement (2005), 111-117.

74. Philip R Ross and Stephan AG Wensveen. 2010. Designing Behavior in Interaction: Using Aesthetic Experience as a Mechanism for Design. International Journal of Design 4, 2 (2010), 3-13.

75. Anne Roudaut, Abhijit Karnik, Markus Löchtefeld, and Sriram Subramanian. 2013. Morphees: Toward High "Shape Resolution" in Self-actuated Flexible Mobile Devices. In Proceedings of the SIGCHI Conference on Human Factors in Computing Systems (CHI '13). ACM, New York, NY, USA, 593-602. DOI : http://dx.doi.org/10.1145/2470654.2470738

76. Anne Roudaut, Diana Krusteva, Mike McCoy, Abhijit Karnik, Karthik Ramani, and Sriram Subramanian. 2016. Cubimorph: Designing Modular Interactive Devices. In 2016 IEEE International Conference on Robotics and Automation (ICRA). 3339-3345. DOI : http://dx.doi.org/10.1109/ICRA.2016.7487508

77. Anne Roudaut, Rebecca Reed, Tianbo Hao, and Sriram Subramanian. 2014. Changibles: Analyzing and Designing Shape Changing Constructive Assembly. In Proceedings of the 32Nd Annual ACM Conference on Human Factors in Computing Systems (CHI'14). ACM, New York, NY, USA, 2593-2596. DOI : http://dx.doi.org/10.1145/2556288.2557006 
78. Daniel Rozin. 2007. Peg Mirror. (2007). Retrieved 15/09/17 from

http://www. smoothware. com/danny/pegmirror.html.

79. Marcos Serrano, Anne Roudaut, and Pourang Irani. 2016. Investigating Text Legibility on Non-Rectangular Displays. In Proceedings of the 2016 CHI Conference on Human Factors in Computing Systems (CHI '16). ACM, New York, NY, USA, 498-508. D0I : http://dx.doi.org/10.1145/2858036.2858057

80. Marcos Serrano, Anne Roudaut, and Pourang Irani. 2017. Visual Composition of Graphical Elements on Non-Rectangular Displays. In Proceedings of the 2017 CHI Conference on Human Factors in Computing Systems (CHI'17). ACM, New York, NY, USA, 4405-4416. DOI : http://dx.doi.org/10.1145/3025453.3025677

81. Orit Shaer and Eva Hornecker. 2010. Tangible User Interfaces: Past, Present, and Future Directions. Found. Trends Hum.-Comput. Interact. 3, 1-2 (Jan. 2010), 1-137. DOI : http://dx.doi .org/10.1561/1100000026

82. Hiroki Shigemune, Shingo Maeda, Vito Cacucciolo, Yoshitaka Iwata, Eiji Iwase, Shujo Hashimoto, and Shigeki Sugano. 2017. Printed Paper Robot Driven by Electrostatic Actuator. IEEE Robotics and Automation Letters 2, 2 (April 2017), 1001-1007. DOI : http://dx.doi.org/10.1109/LRA.2017.2658942

83. Makoto Shimojo, Masami Shinohara, and Yukio Fukui. 1999. Human Shape Recognition Performance for 3D Tactile Display. IEEE Transactions on Systems, Man, and Cybernetics-Part A: Systems and Humans 29, 6 (1999), 637-644.

84. Ben Shneiderman. 1987. Designing the User Interface: Strategies for Effective Human-computer Interaction. Addison-Wesley Longman Publishing Co., Inc., Boston, MA, USA.

85. Adam Spiers, Aaron Dollar, Janet van der Linden, and Maria Oshodi. 2015. First Validation of the Haptic Sandwich: A Shape Changing Handheld Haptic Navigation Aid. In 2015 International Conference on Advanced Robotics (ICAR). 144-151. DOI : http://dx.doi.org/10.1109/ICAR.2015.7251447

86. Mandayam A. Srinivasan and Cagatay Basdogan. 1997. Haptics in Virtual Environments: Taxonomy, Research Status, and Challenges. Computers \& Graphics 21, 4 (1997), 393-404. DOI : http://dx.doi.org/10.1016/S0097-8493(97)00030-7 Haptic Displays in Virtual Environments and Computer Graphics in Korea.

87. Jelle Stienstra, Miguel Bruns Alonso, Stephan Wensveen, and Stoffel Kuenen. 2012. How to Design for Transformation of Behavior Through Interactive Materiality. In Proceedings of the 7th Nordic Conference on Human-Computer Interaction: Making Sense Through Design (NordiCHI '12). ACM, New York, NY, USA, 21-30. DOI : http://dx.doi.org/10.1145/2399016.2399020

88. Evan Strasnick, Jackie Yang, Kesler Tanner, Alex Olwal, and Sean Follmer. 2017. shiftIO: Reconfigurable Tactile
Elements for Dynamic Affordances and Mobile Interaction. In Proceedings of the 2017 CHI Conference on Human Factors in Computing Systems (CHI'17). ACM, New York, NY, USA, 5075-5086. DOI: http://dx.doi.org/10.1145/3025453.3025988

89. Paul Strohmeier, Juan Pablo Carrascal, Bernard Cheng, Margaret Meban, and Roel Vertegaal. 2016. An Evaluation of Shape Changes for Conveying Emotions. In Proceedings of the 2016 CHI Conference on Human Factors in Computing Systems (CHI '16). ACM, New York, NY, USA, 3781-3792. DOI : http://dx.doi.org/10.1145/2858036.2858537

90. ART+COM Studios. 2008. Kinetic Sculpture - The Shapes of Things to Come, 2008. (2008). Retrieved 15/09/17 from https://artcom.de/en/project/kinetic-sculpture/.

91. Miriam Sturdee and Jason Alexander. 2018. Analysis and Classification of Shape-Changing Interfaces for Design and Application-based Research. ACM Comput. Surv. 51, 1, Article 2 (Jan. 2018), 32 pages. DOI :

http://dx.doi.org/10.1145/3143559

92. Miriam Sturdee, Paul Coulton, and Jason Alexander. 2017. Using Design Fiction to Inform Shape-Changing Interface Design and Use. The Design Journal 20, sup1 (2017), 4146-4157. DOI :

http://dx.doi.org/10.1080/14606925.2017.1352913

93. Miriam Sturdee, John Hardy, Nick Dunn, and Jason Alexander. 2015. A Public Ideation of Shape-Changing Applications. In Proceedings of the 2015 International Conference on Interactive Tabletops \& Surfaces (ITS '15). ACM, New York, NY, USA, 219-228. DOI : http://dx.doi.org/10.1145/2817721.2817734

94. Ivan E. Sutherland. 1965. The Ultimate Display. In Proceedings of the IFIP Congress. 506-508.

95. Faisal Taher, John Hardy, Abhijit Karnik, Christian Weichel, Yvonne Jansen, Kasper Hornbæk, and Jason Alexander. 2015. Exploring Interactions with Physically Dynamic Bar Charts. In Proceedings of the 33rd Annual ACM Conference on Human Factors in Computing Systems (CHI '15). ACM, New York, NY, USA, 3237-3246. DOI :

http://dx.doi.org/10.1145/2702123.2702604

96. Faisal Taher, Yvonne Jansen, Jonathan Woodruff, John Hardy, Kasper Hornbæk, and Jason Alexander. 2017a. Investigating the Use of a Dynamic Physical Bar Chart for Data Exploration and Presentation. IEEE Transactions on Visualization and Computer Graphics 23, 1 (Jan 2017), 451-460. DOI :

http://dx.doi.org/10.1109/TVCG.2016.2598498

97. Faisal Taher, John Vidler, and Jason Alexander. 2017b. A Characterization of Actuation Techniques for Generating Movement in Shape-Changing Interfaces. International Journal of Human-Computer Interaction 33, 5 (2017), 385-398. DOI :

http://dx.doi.org/10.1080/10447318.2016.1250372 
98. Haodan Tan, John Tiab, Selma Šabanović, and Kasper Hornbæk. 2016. Happy Moves, Sad Grooves: Using Theories of Biological Motion and Affect to Design Shape-Changing Interfaces. In Proceedings of the 2016 ACM Conference on Designing Interactive Systems (DIS '16). ACM, New York, NY, USA, 1282-1293. DOI : http://dx.doi.org/10.1145/2901790.2901845

99. Seppe Terryn, Joost Brancart, Dirk Lefeber, Guy Van Assche, and Bram Vanderborght. 2017. Self-healing Soft Pneumatic Robots. Science Robotics 2, 9 (2017), eaan4268.

100. John Tiab and Kasper Hornbæk. 2016. Understanding Affordance, System State, and Feedback in Shape-Changing Buttons. In Proceedings of the 2016 CHI Conference on Human Factors in Computing Systems (CHI'16). ACM, New York, NY, USA, 2752-2763. DOI : http://dx.doi.org/10.1145/2858036.2858350

101. Skylar Tibbits. 2014. 4D printing: Multi-material Shape Change. Architectural Design 84, 1 (2014), 116-121.

102. Giovanni Maria Troiano, Esben Warming Pedersen, and Kasper Hornbæk. 2014. User-defined Gestures for Elastic, Deformable Displays. In Proceedings of the 2014 International Working Conference on Advanced Visual Interfaces (AVI '14). ACM, New York, NY, USA, 1-8. DOI : http://dx.doi.org/10.1145/2598153.2598184

103. Philip Tuddenham, David Kirk, and Shahram Izadi. 2010. Graspables Revisited: Multi-touch vs. Tangible Input for Tabletop Displays in Acquisition and Manipulation Tasks. In Proceedings of the SIGCHI Conference on Human Factors in Computing Systems (CHI '10). ACM, New York, NY, USA, 2223-2232. DOI : http://dx.doi.org/10.1145/1753326.1753662

104. Anna Vallgårda, Morten Winther, Nina Mørch, and Edit E Vizer. 2015. Temporal Form in Interaction Design. International Journal of Design 9, 3 (2015), 1-15.
105. Christian Weichel, Jason Alexander, and John Hardy. 2015. Shape Display Shader Language (SDSL): A New Programming Model for Shape Changing Displays. In Proceedings of the 33rd Annual ACM Conference Extended Abstracts on Human Factors in Computing Systems (CHI EA '15). ACM, New York, NY, USA, 1121-1126. DOI :

http://dx.doi.org/10.1145/2702613.2732727

106. Martin Weigel, Tong Lu, Gilles Bailly, Antti Oulasvirta, Carmel Majidi, and Jürgen Steimle. 2015. iSkin: Flexible, Stretchable and Visually Customizable On-Body Touch Sensors for Mobile Computing. In Proceedings of the 33rd Annual ACM Conference on Human Factors in Computing Systems (CHI'15). ACM, New York, NY, USA, 2991-3000. DOI :

http://dx.doi.org/10.1145/2702123.2702391

107. Zheng Yan. 2015. Encyclopedia of Mobile Phone Behavior. IGI Global.

108. Lining Yao, Ryuma Niiyama, Jifei Ou, Sean Follmer, Clark Della Silva, and Hiroshi Ishii. 2013. PneUI: Pneumatically Actuated Soft Composite Materials for Shape Changing Interfaces. In Proceedings of the 26th Annual ACM Symposium on User Interface Software and Technology (UIST '13). ACM, New York, NY, USA, 13-22. DOI : http://dx.doi .org/10.1145/2501988.2502037

109. Mark Yim, Wei-Min Shen, Behnam Salemi, Daniela Rus, Mark Moll, Hod Lipson, Eric Klavins, and Gregory S Chirikjian. 2007. Modular Self-reconfigurable Robot Systems [Grand Challenges of Robotics]. IEEE Robotics \& Automation Magazine 14, 1 (2007), 43-52. DOI : http://dx.doi.org/10.1109/MRA.2007.339623

110. Oren Zuckerman and Ayelet Gal-Oz. 2013. To TUI or not to TUI: Evaluating Performance and Preference in Tangible vs. Graphical User Interfaces. International Journal of Human-Computer Studies 71, 7 (2013), 803 820. DOI :

http://dx.doi.org/10.1016/j.ijhcs.2013.04.003 